\title{
Hydrostatic fluid loading in non-linear finite element analysis
}

\author{
T. Rumpel ${ }^{1}$ and K. Schweizerhof ${ }^{2, *, \dagger}$ \\ ${ }^{1}$ Institut für Technische Mechanik, Universität Karlsruhe (TH), D-76128 Karlsruhe, Germany \\ ${ }^{2}$ Institut für Mechanik, Universität Karlsruhe (TH), Kaiserstrasse 12, D-76128 Karlsruhe, Germany
}

\begin{abstract}
SUMMARY
Deformation dependent pressure loading on solid structures is created by the interaction of fluids with the deformable surface of a structure. Such fairly simple load models are valid for static and quasistatic analyses and they are a very efficient tool to represent the influence of a fluid on the behaviour of structures. Completing previous studies on the deformation dependence of the loading with the assumption of finite gas volumes, the current contribution focuses on the influence of modifications of the size and shape of a finite structure containing an incompressible fluid with a free surface. The linearization of the corresponding virtual work expression necessary for a Newton-type solution leads to additional terms for the volume dependence. Investigating these terms the conservativeness of the problem can be proven by the symmetry of the linearized form. Similarly to gas loading, the discretization of the structure with finite elements leads to standard stiffness matrix forms plus the so-called load stiffness matrices and a rank-one update for each filled structure part, if the loaded surface segments are identical with element surfaces. Some numerical examples show the effectiveness of the approach and the necessity to take the corresponding terms in the variational expression and the following linearization into account. Copyright (c) 2004 John Wiley \& Sons, Ltd.
\end{abstract}

KEY WORDS: hydrostatic fluid loading; volume dependence; large deformations; finite elements

\section{INTRODUCTION}

Pressure loading on structures is usually generated by gas, fluids or wind action and it is mostly considered as static and uniform and its deformation dependence within loading processes is not taken into account. As is well known even for small deformations the stability load of cylinders under external pressure is reduced by a quarter, if the effect is taken into account that the pressure remains normal to the surface [1].

For large deformations further effects appear such as the extension of pressure loaded surfaces and their motion within a loading field such as in water, see References $[2,3]$ and the papers based on them [4-8]. Then it is rather important to consider the current position of the loaded part with respect to the fluid surface to get the correct loading. If enclosed volumes are

\footnotetext{
*Correspondence to: K. Schweizerhof, Institut für Mechanik, Universität Karlsruhe (TH), Kaiserstrasse 12, D-76128 Karlsruhe, Germany.

†E-mail: karl.schweizerhof@bau-verm.uni-karlsruhe.de
}

Copyright (c) 2004 John Wiley \& Sons, Ltd.

Received 25 October 2002

Revised 21 May 2003

Accepted 22 May 2003 
considered such as tires or airbags then the volume change within the loading process has a major effect on the local pressure. This was presented in detail in Reference [9]. Similar problems occur if the structures show large deformations and contain fluids [5,8], and are otherwise loaded, such as water bags or tubes. In general, volume effects are more important for membrane-type structures respectively thin flexible shell structures. Besides the recent study by Bonet et al. [10] and in Reference [9] volume effects have been mainly taken into account in fluid structure analysis with complete FE models for the fluid and the structure parts.

Within the following our focus is on hydrostatic fluid loading and on the corresponding derivations for static implicit solution schemes to allow large deformation FE analyses. Specifically, the conditions for conservativeness of the problems are also investigated.

\section{THE GOVERNING EQUATIONS}

Assuming that we describe the general non-linear structural behaviour in a standard fashion our focus is only on the external virtual work of a pressure load for the structure part and on the additional equations specifying the physical behaviour of the fluid.

\subsection{External virtual work of pressure loading acting on a given structure}

The deformation of a pressurized structural part — containing a fluid — can be described within the framework of virtual work via the external virtual work of a pressure load as shown in References [11-13]. The pressure level $p$ is then dependent on the current position $\mathbf{x}$ of all surface points of the structure in space and their current position relative to the free fluid surface.

The external virtual work becomes

$$
\delta \Pi_{\text {press }}=\int_{a} p \mathbf{n} \cdot \delta \mathbf{u} \mathrm{d} a
$$

with the normal $\mathbf{n}=\mathbf{x}, \xi \times \mathbf{x}, \eta /|\mathbf{x}, \xi \times \mathbf{x}, \eta|$, the virtual displacement $\delta \mathbf{u}$, the surface element $\mathrm{d} a=|\mathbf{x}, \xi \times \mathbf{x}, \eta| \mathrm{d} \xi \mathrm{d} \eta$ of the wetted surface $a$ and the pressure level $p$. The position vector $\mathbf{x}(\xi, \eta)$ depends on local co-ordinates $\xi, \eta$, see Figure 1 . Thus the pressure is acting perpendicular to the surface of the structure; the surface $\mathrm{d} a$ may be modified under loading in size and in direction, the latter represented by $\mathbf{n}$. To simplify matters a non-normalized form $\mathbf{n}^{*}=\mathbf{x}, \xi \times \mathbf{x}, \eta$ of the normal vector $\mathbf{n}$ is introduced, resulting in

$$
\delta \Pi_{\text {press }}=\int_{\eta} \int_{\xi} p(\mathbf{x}, \xi \times \mathbf{x}, \eta) \cdot \delta \mathbf{u} \mathrm{d} \xi \mathrm{d} \eta=\int_{\eta} \int_{\xi} p \mathbf{n}^{*} \cdot \delta \mathbf{u} \mathrm{d} \xi \mathrm{d} \eta
$$

\subsection{Constitutive equations of a fluid filled structure with a free fluid surface}

The pressure distribution in a fluid filled structure, which has several chambers/containers $i$, is described by the hydrostatic pressure law (3) and the volume conservation condition (4) of the contained fluid-assuming incompressibility

$$
\begin{aligned}
p_{i} & =\gamma_{i}\left(\mathbf{x}_{w i}-\mathbf{x}_{i}\right) \cdot \mathbf{w} \\
v_{i} & =\text { const. }
\end{aligned}
$$




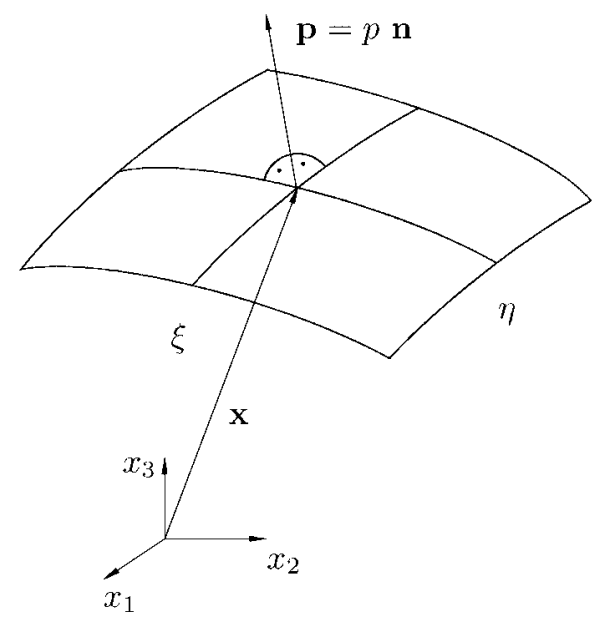

Figure 1. Surface under pressure loading.
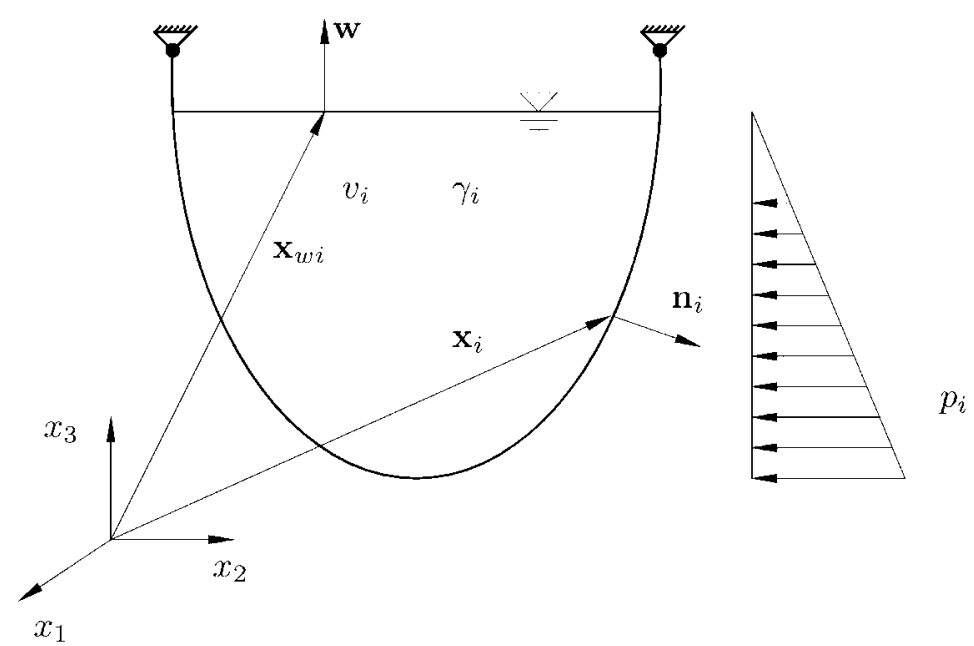

Figure 2. Hydrostatic pressure loading; volume computation of fluid filled structures.

with $\gamma_{i}=\rho_{i} g$ is the specific gravity, $\rho_{i}$ the specific density of the fluid, $g$ the acceleration due to gravity, $\mathbf{x}_{w i}$ the position vector to the fluid surface in container $i$ and $\mathbf{w}$ the unit normal vector on the fluid surface, see Figure 2. It must be noted that $\mathbf{w}$ is identical for all containers. The volume $v_{i}$ of the fluid in one container $i$ can then be computed via

$$
v_{i}=\frac{1}{3} \int_{\eta} \int_{\xi}\left(\mathbf{x}_{i} \cdot \mathbf{n}_{i}^{*}+\mathbf{x}_{w i} \cdot \mathbf{w}_{i}^{*}\right) \mathrm{d} \xi \mathrm{d} \eta
$$

Corresponding to the non-normalized normal vector $\mathbf{n}_{i}^{*}$ on the wetted surface of the container $i$, $\mathbf{w}_{i}^{*}=\mathbf{x}_{w i, \xi} \times \mathbf{x}_{w i, \eta}$ denotes the non-normalized normal vector of the free fluid surface. Thus the 


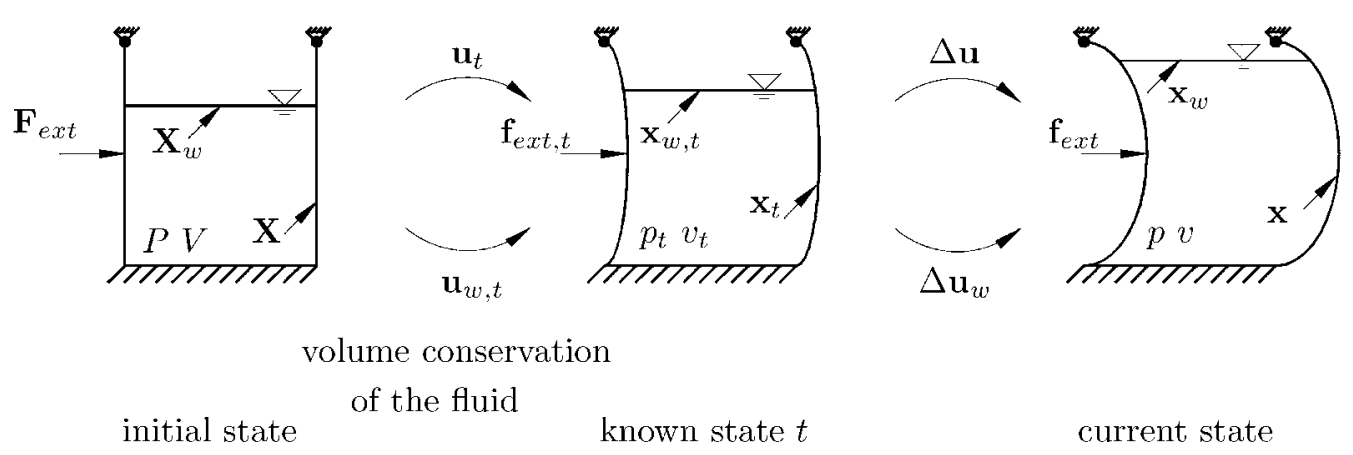

Figure 3. States within a Newton scheme for hydrostatic pressure load.

major difference to a closed volume is simply the fact that together with the wetted surface of the structure the fluid surface describes the closure of the considered fluid volume. In the following the index $i$ is omitted for reasons of brevity.

\section{LINEARIZATION OF THE GOVERNING EQUATIONS}

If we consider a description of the structure with shell, volume or beam element surfaces and assume non-linear behaviour, the system of non-linear equations resulting from the variational expression must be linearized for the solution with a Newton-type scheme. Then the linearization at state $t$ has to be performed before the discretization with, e.g. finite elements and within an incremental iterative procedure.

\subsection{Linearization of the virtual work expression}

The necessary quantities are $\mathbf{X}$ as initial position of a structural surface point, $\mathbf{x}_{t}=\mathbf{X}+\mathbf{u}_{t}$ as known position at state $t$ and therefore $\mathbf{x}=\mathbf{x}_{t}+\Delta \mathbf{u}$ as position at the current state of the structure, see Figure 3. Following the chain rule the different contributions have to be linearized separately. The expression for the external virtual work of the considered volume including the linear part of the changes of the normal which is integrated over the wetted surface of the structure can be written as

$$
\begin{aligned}
& \delta \Pi_{\text {press }}^{\mathrm{lin}}=\delta \Pi_{\text {press }, t}+\delta \Pi_{\text {press }, t}^{\Delta p}+\delta \Pi_{\text {press }, t}^{\Delta \mathbf{n}} \\
& \delta \Pi_{\text {press }}^{\operatorname{lin}}=\int_{\eta} \int_{\xi}\left(p_{t} \mathbf{n}_{t}^{*} \cdot \delta \mathbf{u}+\Delta p \mathbf{n}_{t}^{*} \cdot \delta \mathbf{u}+p_{t} \Delta \mathbf{n}^{*} \cdot \delta \mathbf{u}\right) \mathrm{d} \xi \mathrm{d} \eta
\end{aligned}
$$

with

$$
\Delta \mathbf{n}^{*}=\Delta \mathbf{u}, \xi \times \mathbf{x}_{t, \eta}-\Delta \mathbf{u}, \eta \times \mathbf{x}_{t}, \xi
$$

for the change of the non-normalized linearized normal vector. $P\left(\mathbf{X}_{w}, \mathbf{X}\right), V$ denote the initial pressure and volume, $p_{t}=p_{t}\left(\mathbf{x}_{w}, \mathbf{x}\right), v_{t}$ are the known pressure and volume, respectively. 


\subsection{Linearization of the hydrostatic pressure law and of the volume conservation condition}

The linearized hydrostatic pressure and the volume conservation condition can be written as

$$
\begin{aligned}
\Delta p & =\gamma\left(\Delta \mathbf{u}_{w}-\Delta \mathbf{u}\right) \cdot \mathbf{w} \\
\Delta v & =\frac{1}{3} \int_{\eta} \int_{\xi}\left(\Delta \mathbf{u} \cdot \mathbf{n}_{t}^{*}+\mathbf{x}_{t} \cdot \Delta \mathbf{n}^{*}+\Delta \mathbf{u}_{w} \cdot \mathbf{w}_{t}^{*}+\mathbf{x}_{w, t} \cdot \Delta \mathbf{w}^{*}\right) \mathrm{d} \xi \mathrm{d} \eta=0 \\
& \equiv \Delta v^{\Delta \mathbf{u}}+\Delta v^{\Delta \mathbf{n}}+\Delta v^{\Delta \mathbf{u}_{w}}+\Delta v^{\Delta \mathbf{w}}=0
\end{aligned}
$$

Where $\mathbf{x}_{w}=\mathbf{x}_{w, t}+\Delta \mathbf{u}_{w}$ is the current fluid level and $\Delta \mathbf{w}^{*}$ the change of the non-normalized normal vector of the free fluid surface, see Figure 3 . The volume change-in total zerocan be expressed as shown in Equations (10) and (11) by the volume change due to the displacement of the structure $\Delta v^{\Delta \mathbf{u}}$ and the fluid level $\Delta v^{\Delta \mathbf{u}_{w}}$ and the volume change due to the change of the normals on the wetted structure $\Delta v^{\Delta \mathbf{n}}$ and the free fluid surface $\Delta v^{\Delta \mathbf{w}}$.

\section{CONSERVATIVENESS AND SYMMETRIC MATRICES}

It is known that the discussion of the conservativeness of the considered problem taking into account large deformations can be reduced to the discussion of the symmetry of the linearized expression concerning the displacements and the virtual displacements, see References [1, 14, 15] for uniform pressure, [2,3] for non-uniform pressure fields and [4] for a summary based on Reference [2]. Within the following we extend these considerations to the above introduced problems, see also References $[7,9,11,13,16]$. If partial integration is used in a special fashion, the linearized expression of the external virtual work and the additional equations especially the volume change due to the change of the normals can be rewritten.

\subsection{Partial integration of the external virtual work-considering the change of the normal}

First our focus on the partial integration of the external virtual work is restricted to the virtual work expression $\delta \Pi_{\text {press, } t}^{\Delta \mathbf{n}}$, see Equations (6), (7), which is a function of the linearized non-normalized normal vector $\Delta \mathbf{n}^{*}$. Introducing the covariant base vectors $\mathbf{g}_{\xi}=\mathbf{x}_{t, \xi}, \mathbf{g}_{\eta}=\mathbf{x}_{t, \eta}$, which denote also the components of the boundary normal $\mathbf{n}_{t}^{s}$, leads for the virtual work expression to

$$
\begin{aligned}
\delta \Pi_{\mathrm{press}, t}^{\Delta \mathbf{n}} & =\int_{\eta} \int_{\xi} p_{t} \Delta \mathbf{n}^{*} \cdot \delta \mathbf{u} \mathrm{d} \xi \mathrm{d} \eta \\
& =\int_{\eta} \int_{\xi} p_{t}\left[\left(\mathbf{g}_{\eta} \times \delta \mathbf{u}\right) \cdot \Delta \mathbf{u}_{, \xi}-\left(\mathbf{g}_{\xi} \times \delta \mathbf{u}\right) \cdot \Delta \mathbf{u}_{, \eta}\right] \mathrm{d} \xi \mathrm{d} \eta \\
& =\int_{s} p_{t}^{s}\left(n_{\xi} \mathbf{g}_{\eta} \times \delta \mathbf{u}^{s}-n_{\eta}^{s} \mathbf{g}_{\xi} \times \delta \mathbf{u}^{s}\right) \cdot \Delta \mathbf{u}^{s} \mathrm{~d} s
\end{aligned}
$$




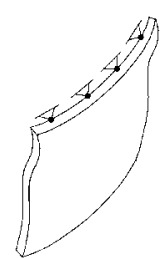

$\mathrm{C} 1$

$\Delta \mathbf{u}^{s}=0$

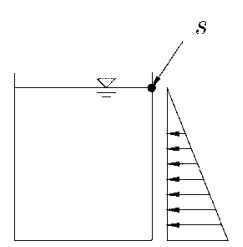

$\mathrm{C} 2$

$p_{t}^{s}=0$

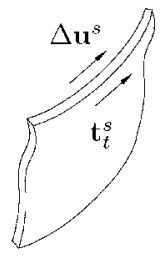

$\mathrm{C} 3 \mathrm{a}$



$\mathrm{C} 3 \mathrm{~b}$

Figure 4. Boundary conditions leading to the disappearance of the boundary integral.

$$
\begin{aligned}
& -\int_{\eta} \int_{\xi} p_{t}\left(\mathbf{g}_{\eta} \times \delta \mathbf{u}_{, \xi}-\mathbf{g}_{\xi} \times \delta \mathbf{u}_{, \eta}\right) \cdot \Delta \mathbf{u} \mathrm{d} \xi \mathrm{d} \eta \\
& -\int_{\eta} \int_{\xi}\left[p_{t, \xi}\left(\mathbf{g}_{\eta} \times \delta \mathbf{u}\right)-p_{t, \eta}\left(\mathbf{g}_{\xi} \times \delta \mathbf{u}\right)\right] \cdot \Delta \mathbf{u} \mathrm{d} \xi \mathrm{d} \eta
\end{aligned}
$$

Assuming for illustration purposes a cartesian co-ordinate system at the boundary $s$ the normal vector $\mathbf{n}_{t}^{s}$ and the tangent vector $\mathbf{t}_{t}^{s}$ can be written with $n_{\xi}^{s}, n_{\eta}^{s}$ as co-ordinates of the covariant system as

$$
\left(\begin{array}{c}
\mathbf{n}_{t}^{s} \\
\mathbf{t}_{t}^{s}
\end{array}\right)=\left[\begin{array}{cc}
n_{\xi}^{s} & n_{\eta}^{s} \\
-n_{\eta}^{s} & n_{\xi}^{s}
\end{array}\right]\left(\begin{array}{l}
\mathbf{g}_{\xi} \\
\mathbf{g}_{\eta}
\end{array}\right)
$$

The relevant part of the external virtual work can then be reduced to

$$
\begin{aligned}
\delta \Pi_{\text {press }, t}^{\Delta \mathbf{n}}= & \int_{s} p_{t}^{s}\left(\Delta \mathbf{u}^{s} \times \mathbf{t}_{t}^{s}\right) \cdot \delta \mathbf{u}^{s} \mathrm{~d} s \\
& -\int_{\eta} \int_{\xi} p_{t}\left(\mathbf{g}_{\eta} \times \delta \mathbf{u}_{, \xi}-\mathbf{g}_{\xi} \times \delta \mathbf{u}_{, \eta}\right) \cdot \Delta \mathbf{u} \mathrm{d} \xi \mathrm{d} \eta \\
& -\int_{\eta} \int_{\xi}\left[p_{t, \xi}\left(\mathbf{g}_{\eta} \times \delta \mathbf{u}\right)-p_{t, \eta}\left(\mathbf{g}_{\xi} \times \delta \mathbf{u}\right)\right] \cdot \Delta \mathbf{u} \mathrm{d} \xi \mathrm{d} \eta
\end{aligned}
$$

As already shown in References [2, 3,9,11,16,17] the boundary integral vanishes - the first term in Equation (16) - if one of the following five conditions is valid, see also Figure 4.

C1. Fixed boundary: $\Delta \mathbf{u}^{s}=0$

C2. No loading $p$ on complete boundary $s: p_{t}^{s}=0$. This is valid for the surface boundary of structures which are not completely filled with fluid.

C3. For any boundary with $p \neq 0$, p.e. pressurized gas on the top of the fluid.

(a) Only displacements parallel to the considered boundary $s$ are allowed: $\Delta \mathbf{u}^{s} \| \mathbf{t}_{t}^{s} \rightarrow$ $\Delta \mathbf{u}^{s} \times \mathbf{t}_{t}^{s}=\mathbf{0}$. This is physically clear as $\mathbf{t}_{t}^{s}$ is perpendicular to $p_{t}^{S} \mathbf{n}_{t}^{s}$ and the virtual work expression vanishes. 
(b) One boundary displacement component perpendicular to the tangent vector is fixed: $\left(\Delta \mathbf{u}^{s} \times \mathbf{t}_{t}^{s}\right) \cdot \delta \mathbf{u}^{s}=0$. This corrects the statements given in References $[2,3,16,17]$ and by others. Typical for sliding boundary conditions.

C4. There is no boundary, thus only closed structures completely filled with gas or fluid are considered $[9,11,17]$.

C5. The boundary integral vanishes as a whole, which is hard to interpret physically, if there are no distinct boundary conditions.

For further conversions, see References $[2,3,9,16]$, contravariant base vectors $\mathbf{g}^{\xi}, \mathbf{g}^{\eta}$ are introduced:

$$
-\mathbf{g}_{\xi}=\mathbf{n}^{*} \times \mathbf{g}^{\eta}, \quad \mathbf{g}_{\eta}=\mathbf{n}^{*} \times \mathbf{g}^{\xi} \quad \text { with } \quad \mathbf{n}^{*}=\mathbf{g}_{\xi} \times \mathbf{g}_{\eta}
$$

Introducing skew symmetric tensors:

$$
\begin{aligned}
& \underline{\mathbf{W}}^{\xi}=\mathbf{n}^{*} \otimes \mathbf{g}^{\xi}-\mathbf{g}^{\xi} \otimes \mathbf{n}^{*} \\
& \underline{\mathbf{W}}^{\eta}=\mathbf{n}^{*} \otimes \mathbf{g}^{\eta}-\mathbf{g}^{\eta} \otimes \mathbf{n}^{*}
\end{aligned}
$$

the relevant part of the external virtual work expression becomes then

$$
\begin{aligned}
\delta \Pi_{\text {press }, t}^{\Delta \mathbf{n}}= & -\frac{1}{2} \int_{\eta} \int_{\xi}\left[p_{t, \xi} \delta \mathbf{u} \cdot\left(\mathbf{n}^{*} \otimes \mathbf{g}^{\xi}-\mathbf{g}^{\xi} \otimes \mathbf{n}^{*}\right) \Delta \mathbf{u}\right. \\
& \left.+p_{t, \eta} \delta \mathbf{u} \cdot\left(\mathbf{n}^{*} \otimes \mathbf{g}^{\eta}-\mathbf{g}^{\eta} \otimes \mathbf{n}^{*}\right) \Delta \mathbf{u}\right] \mathrm{d} \xi \mathrm{d} \eta \\
& +\int_{\eta} \int_{\xi} \frac{p_{t}}{2}\left(\begin{array}{c}
\delta \mathbf{u} \\
\delta \mathbf{u}, \xi \\
\delta \mathbf{u}, \eta
\end{array}\right) \cdot\left[\begin{array}{ccc}
0 & \underline{\mathbf{W}^{\xi}} & \underline{\mathbf{W}}^{\eta} \\
\underline{\mathbf{W}}^{\xi \mathrm{T}} & 0 & 0 \\
\underline{\mathbf{W}}^{\eta \mathrm{T}} & 0 & 0
\end{array}\right]\left(\begin{array}{c}
\Delta \mathbf{u} \\
\Delta \mathbf{u}, \xi \\
\Delta \mathbf{u}, \boldsymbol{\eta}
\end{array}\right) \mathrm{d} \xi \mathrm{d} \eta
\end{aligned}
$$

For details of the derivation we refer to References $[9,16]$.

4.2. Partial integration of the volume conservation for a fluid filled structure-considering the change of the normal

As presented in Section 4.1 the partial integration of the volume conservation focusses on the change of the normal $\mathbf{n}^{*}$ of the structure and consequently also on the change of the normal $\mathbf{w}^{*}$ of the free fluid level, see also the transformations given in the above section

$$
\begin{aligned}
\Delta v^{\Delta \mathbf{n}}+\Delta v^{\Delta \mathbf{w}}= & \frac{1}{3} \int_{\eta} \int_{\xi}\left(\mathbf{x}_{t} \cdot \Delta \mathbf{n}^{*}+\mathbf{x}_{w, t} \cdot \Delta \mathbf{w}^{*}\right) \mathrm{d} \xi \mathrm{d} \eta \\
= & \frac{1}{3} \int_{s}\left[\left(\Delta \mathbf{u} \times \mathbf{t}_{t}^{s}\right) \cdot \mathbf{x}_{t}+\left(\Delta \mathbf{u}_{w} \times \mathbf{t}_{w, t}^{s}\right) \cdot \mathbf{x}_{w, t}\right] \mathrm{d} s \\
& +\frac{2}{3} \int_{\eta} \int_{\xi}\left(\mathbf{n}_{t}^{*} \cdot \Delta \mathbf{u}+\mathbf{w}_{t}^{*} \cdot \Delta \mathbf{u}_{w}\right) \mathrm{d} \xi \mathrm{d} \eta
\end{aligned}
$$


At the boundary of the fluid on the wetted structure the position vector $\mathbf{x}_{t}$ of the structure is equal to the position vector $\mathbf{x}_{w, t}$ and therefore the displacement and the tangent vector become: $\mathbf{u}=\mathbf{u}_{w}$ and $\mathbf{t}_{t}^{s}=-\mathbf{t}_{w, t}^{s}$ respectively. Thus the boundary integral vanishes as a whole. The volume conservation then results after the addition of the other parts $\Delta v^{\Delta \mathbf{u}}$ and $\Delta v^{\Delta \mathbf{u} w}$ from Equation (10) in

$$
\Delta v=\int_{\eta} \int_{\xi}\left(\mathbf{n}_{t}^{*} \cdot \Delta \mathbf{u}+\mathbf{w}_{t}^{*} \cdot \Delta \mathbf{u}_{w}\right) \mathrm{d} \xi \mathrm{d} \eta=0
$$

The last part of Equation (23) expresses the volume change as a result of the fluid level displacement $\Delta v^{\Delta \mathbf{u}_{w}}$. This part of the integral can be rewritten using the unit normal vector $\mathbf{w}$ of the free fluid surface area $S_{t}$. The area $S_{t}$ of the free fluid surface can be computed via a projection of the wetted surface of the structure into the direction of the free fluid surface normal w, see also Equations (26)-(28)

$$
S_{t}=\int_{\eta} \int_{\xi} \mathbf{n}_{t}^{*} \cdot \mathbf{w} \mathrm{d} \xi \mathrm{d} \eta
$$

In order to get a correct surface computation the orientation of the structure surface-thus the corresponding normal- has to be consistent for the complete wetted part. As the displacement $\Delta \mathbf{u}_{w}$ of the free fluid surface has only a component in $\mathbf{w}$-direction, it can be expressed as a scalar $\Delta u_{w}=\Delta \mathbf{u}_{w} \cdot \mathbf{w}$ and the volume change due to fluid level displacement as

$$
\begin{aligned}
\Delta v^{\Delta \mathbf{u}_{w}} & =\int_{\eta} \int_{\xi} \mathbf{w}_{t}^{*} \cdot \Delta \mathbf{u}_{w} \mathrm{~d} \xi \mathrm{d} \eta \\
& =\Delta u_{w} \int_{\eta} \int_{\xi} \mathbf{w}_{t}^{*} \cdot \mathbf{w} \mathrm{d} \xi \mathrm{d} \eta \\
& =-\Delta u_{w} \int_{\eta} \int_{\xi} \mathbf{n}_{t}^{*} \cdot \mathbf{w} \mathrm{d} \xi \mathrm{d} \eta \\
& =-\Delta u_{w} S_{t}
\end{aligned}
$$

Thus the volume conservation condition of Equation (23) allows to express the displacement of the fluid level as a function of the displacements of the wetted structure

$$
\Delta u_{w} S_{t}=\int_{\eta} \int_{\xi} \mathbf{n}_{t}^{*} \cdot \Delta \mathbf{u} \mathrm{d} \xi \mathrm{d} \eta
$$

Introducing Equation (29) into Equation (9) allows the description of the pressure change via the displacements of the wetted structure

$$
\Delta p=\frac{\gamma}{S_{t}} \int_{\eta} \int_{\xi} \mathbf{n}_{t}^{*} \cdot \Delta \mathbf{u} \mathrm{d} \xi \mathrm{d} \eta-\gamma \mathbf{w} \cdot \Delta \mathbf{u}
$$

As a consequence of Equation (30) the virtual work expression $\delta \Pi_{\mathrm{press}, t}^{\Delta p}$ can be rewritten

$$
\delta \Pi_{\text {press }, t}^{\Delta p}=\frac{\gamma}{S_{t}} \int_{\eta} \int_{\xi} \delta \mathbf{u} \cdot \mathbf{n}_{t}^{*} \mathrm{~d} \xi \mathrm{d} \eta \int_{\eta} \int_{\xi} \mathbf{n}_{t}^{*} \cdot \Delta \mathbf{u} \mathrm{d} \xi \mathrm{d} \eta-\gamma \int_{\eta} \int_{\xi} \delta \mathbf{u} \cdot \mathbf{n}_{t}^{*} \mathbf{w} \cdot \Delta \mathbf{u} \mathrm{d} \xi \mathrm{d} \eta
$$


It can be seen that the first part of Equation (31) has already a symmetric form, showing the latter also for the system 'stiffness' matrix.

\subsection{Symmetric tangent matrices}

Considering the complete linearization, we sum up the separately considered parts in Sections 4.1 and 4.2 .

$$
\begin{gathered}
\delta \Pi_{\text {press }}^{\operatorname{lin}}=0 \\
\delta \Pi_{\text {press }, t}^{\Delta p}+\delta \Pi_{\text {press }, t}^{\Delta \mathbf{n}}=-\delta \Pi_{\text {press }, t}
\end{gathered}
$$

Further conversions are necessary to show the symmetric form of the stiffness matrix.

$$
\begin{aligned}
& \frac{\gamma}{S_{t}} \int_{\eta} \int_{\xi} \delta \mathbf{u} \cdot \mathbf{n}_{t}^{*} \mathrm{~d} \xi \mathrm{d} \eta \int_{\eta} \int_{\xi} \mathbf{n}_{t}^{*} \cdot \Delta \mathbf{u} \mathrm{d} \xi \mathrm{d} \eta \quad \text { (volume conservation I) } \\
& -\gamma \int_{\eta} \int_{\xi} \delta \mathbf{u} \cdot \mathbf{n}_{t}^{*} \mathbf{w} \cdot \Delta \mathbf{u} \mathrm{d} \xi \mathrm{d} \eta \quad \text { (volume conservation II) } \\
& -\frac{1}{2} \int_{\eta} \int_{\xi}\left[p_{t, \xi} \delta \mathbf{u} \cdot\left(\mathbf{n}_{t}^{*} \otimes \mathbf{g}^{\xi}-\mathbf{g}^{\xi} \otimes \mathbf{n}_{t}^{*}\right) \Delta \mathbf{u}\right. \\
& \left.+p_{t, \eta} \delta \mathbf{u} \cdot\left(\mathbf{n}_{t}^{*} \otimes \mathbf{g}^{\eta}-\mathbf{g}^{\eta} \otimes \mathbf{n}_{t}^{*}\right) \Delta \mathbf{u}\right] \mathrm{d} \xi \mathrm{d} \eta \\
& \text { (change of local pressure due to hydrostatic pressure) } \\
& +\frac{1}{2} \int_{\eta} \int_{\xi} p_{t}\left(\begin{array}{c}
\delta \mathbf{u} \\
\delta \mathbf{u}, \xi \\
\delta \mathbf{u}, \eta
\end{array}\right) \cdot\left[\begin{array}{ccc}
0 & \underline{\mathbf{W}}^{\xi} & \underline{\mathbf{W}}^{\eta} \\
\underline{\mathbf{W}}^{\xi \mathrm{T}} & 0 & 0 \\
\underline{\mathbf{W}}^{\eta \mathrm{T}} & 0 & 0
\end{array}\right]\left(\begin{array}{c}
\Delta \mathbf{u} \\
\Delta \mathbf{u}, \xi \\
\Delta \mathbf{u}, \boldsymbol{\eta}
\end{array}\right) \mathrm{d} \xi \mathrm{d} \eta \\
& \text { (normal change and structural displacements) } \\
& =-\int_{\eta} \int_{\xi} p_{t} \delta \mathbf{u} \cdot \mathbf{n}_{t}^{*} \mathrm{~d} \xi \mathrm{d} \eta \quad(\text { load vector })
\end{aligned}
$$

Besides the second part of the volume conservation and the hydrostatic pressure part all other parts indicate symmetric forms. Further conversions have to be performed to show also a symmetric form for these parts $\left(\delta \Pi_{\text {press }, t}^{\circ}\right)$. Summing up the corresponding two terms we obtain for one fluid filled container:

$$
\begin{aligned}
\delta \Pi_{\mathrm{press}, t}^{\circ}= & -\gamma \int_{\eta} \int_{\xi} \delta \mathbf{u} \cdot \mathbf{n}_{t}^{*} \mathbf{w} \cdot \Delta \mathbf{u} \mathrm{d} \xi \mathrm{d} \eta \\
& -\frac{1}{2} \int_{\eta} \int_{\xi}\left[p_{t, \xi} \delta \mathbf{u} \cdot\left(\mathbf{n}_{t}^{*} \otimes \mathbf{g}^{\xi}-\mathbf{g}^{\xi} \otimes \mathbf{n}_{t}^{*}\right) \Delta \mathbf{u}\right. \\
& \left.+p_{t, \eta} \delta \mathbf{u} \cdot\left(\mathbf{n}_{t}^{*} \otimes \mathbf{g}^{\eta}-\mathbf{g}^{\eta} \otimes \mathbf{n}_{t}^{*}\right) \Delta \mathbf{u}\right] \mathrm{d} \xi \mathrm{d} \eta
\end{aligned}
$$


The spatial pressure gradient can be written as

$$
p_{t, \xi}=-\gamma \mathbf{g}_{\xi} \cdot \mathbf{w}, \quad p_{t, \eta}=-\gamma \mathbf{g}_{\eta} \cdot \mathbf{w}
$$

The remaining part of the virtual work expression becomes

$$
\begin{aligned}
\delta \Pi_{\mathrm{press}, t}^{\circ}= & -\frac{\gamma}{2} \int_{\eta} \int_{\xi} \delta \mathbf{u} \cdot\left[2 \mathbf{n}_{t}^{*} \otimes \mathbf{w}-\mathbf{g}_{\xi} \cdot \mathbf{w}\left(\mathbf{n}_{t}^{*} \otimes \mathbf{g}^{\xi}-\mathbf{g}^{\xi} \otimes \mathbf{n}_{t}^{*}\right)\right. \\
& \left.-\mathbf{g}_{\eta} \cdot \mathbf{w}\left(\mathbf{n}_{t}^{*} \otimes \mathbf{g}^{\eta}-\mathbf{g}^{\eta} \otimes \mathbf{n}_{t}^{*}\right)\right] \Delta \mathbf{u} \mathrm{d} \xi \mathrm{d} \eta
\end{aligned}
$$

The unsymmetric tensor $2 \mathbf{n}_{t}^{*} \otimes \mathbf{w}$ can be split into a symmetric and a skew symmetric part

$$
2 \underbrace{\mathbf{n}_{t}^{*} \otimes \mathbf{w}}_{\text {unsym. }}=\underbrace{\mathbf{n}_{t}^{*} \otimes \mathbf{w}+\mathbf{w} \otimes \mathbf{n}_{t}^{*}}_{\text {sym. }}+\underbrace{\mathbf{n}_{t}^{*} \otimes \mathbf{w}-\mathbf{w} \otimes \mathbf{n}_{t}^{*}}_{\text {skew }}
$$

The introduction of Equation (38) into the relevant part of the virtual work expression leads to

$$
\begin{aligned}
\delta \Pi_{\mathrm{press}, t}^{\circ}= & -\frac{\gamma}{2} \int_{\eta} \int_{\xi} \delta \mathbf{u} \cdot\left[\mathbf{n}_{t}^{*} \otimes \mathbf{w}+\mathbf{w} \otimes \mathbf{n}_{t}^{*}+\mathbf{n}_{t}^{*} \otimes \mathbf{w}-\mathbf{w} \otimes \mathbf{n}_{t}^{*}\right. \\
& \left.-\mathbf{g}_{\xi} \cdot \mathbf{w}\left(\mathbf{n}_{t}^{*} \otimes \mathbf{g}^{\xi}-\mathbf{g}^{\xi} \otimes \mathbf{n}_{t}^{*}\right)-\mathbf{g}_{\eta} \cdot \mathbf{w}\left(\mathbf{n}_{t}^{*} \otimes \mathbf{g}^{\eta}-\mathbf{g}^{\eta} \otimes \mathbf{n}_{t}^{*}\right)\right] \Delta \mathbf{u} \mathrm{d} \xi \mathrm{d} \eta
\end{aligned}
$$

Adding to the above expression the following vanishing term, see also Reference [7]:

$$
-\mathbf{g}_{\zeta} \cdot \mathbf{w}\left(\mathbf{n}_{t}^{*} \otimes \mathbf{g}^{\zeta}-\mathbf{g}^{\zeta} \otimes \mathbf{n}_{t}^{*}\right)=0
$$

the remaining part of the virtual work expression can be rewritten again

$$
\begin{aligned}
\delta \Pi_{\mathrm{press}, t}^{\circ}= & -\frac{\gamma}{2} \int_{\eta} \int_{\xi} \delta \mathbf{u} \cdot\left[\mathbf{n}_{t}^{*} \otimes \mathbf{w}+\mathbf{w} \otimes \mathbf{n}_{t}^{*}+\mathbf{n}_{t}^{*} \otimes \mathbf{w}-\mathbf{w} \otimes \mathbf{n}_{t}^{*}\right. \\
& -\mathbf{g}_{\xi} \cdot \mathbf{w}\left(\mathbf{n}_{t}^{*} \otimes \mathbf{g}^{\xi}-\mathbf{g}^{\xi} \otimes \mathbf{n}_{t}^{*}\right) \\
& -\mathbf{g}_{\eta} \cdot \mathbf{w}\left(\mathbf{n}_{t}^{*} \otimes \mathbf{g}^{\eta}-\mathbf{g}^{\eta} \otimes \mathbf{n}_{t}^{*}\right) \\
& \left.-\mathbf{g}_{\zeta} \cdot \mathbf{w}\left(\mathbf{n}_{t}^{*} \otimes \mathbf{g}^{\zeta}-\mathbf{g}^{\zeta} \otimes \mathbf{n}_{t}^{*}\right)\right] \Delta \mathbf{u} \mathrm{d} \xi \mathrm{d} \eta \\
= & -\frac{\gamma}{2} \int_{\eta} \int_{\xi} \delta \mathbf{u} \cdot\left[\mathbf{n}_{t}^{*} \otimes \mathbf{w}+\mathbf{w} \otimes \mathbf{n}_{t}^{*}+\mathbf{n}_{t}^{*} \otimes \mathbf{w}-\mathbf{w} \otimes \mathbf{n}_{t}^{*}\right. \\
& -\mathbf{g}_{\xi} \otimes \mathbf{g}^{\xi} \mathbf{n}_{t}^{*} \otimes \mathbf{w}+\mathbf{g}_{\xi} \otimes \mathbf{g}^{\xi} \mathbf{w} \otimes \mathbf{n}_{t}^{*} \\
& -\mathbf{g}_{\eta} \otimes \mathbf{g}^{\eta} \mathbf{n}_{t}^{*} \otimes \mathbf{w}+\mathbf{g}_{\eta} \otimes \mathbf{g}^{\eta} \mathbf{w} \otimes \mathbf{n}_{t}^{*} \\
& \left.-\mathbf{g}_{\zeta} \otimes \mathbf{g}^{\zeta} \mathbf{n}_{t}^{*} \otimes \mathbf{w}+\mathbf{g}_{\zeta} \otimes \mathbf{g}^{\zeta} \mathbf{w} \otimes \mathbf{n}_{t}^{*}\right] \Delta \mathbf{u d} \mathrm{d} \mathrm{d} \eta
\end{aligned}
$$


Further conversions using $\underline{\mathbf{I}}=\mathbf{g}_{\xi} \otimes \mathbf{g}^{\xi}+\mathbf{g}_{\eta} \otimes \mathbf{g}^{\eta}+\mathbf{g}_{\zeta} \otimes \mathbf{g}^{\zeta}$ show that the unsymmetric part vanishes completely

$$
\begin{aligned}
\delta \Pi_{\mathrm{press}, t}^{\circ}= & -\frac{\gamma}{2} \int_{\eta} \int_{\xi} \delta \mathbf{u} \cdot\left(\mathbf{n}_{t}^{*} \otimes \mathbf{w}+\mathbf{w} \otimes \mathbf{n}_{t}^{*}\right. \\
& \left.+\mathbf{n}_{t}^{*} \otimes \mathbf{w}-\mathbf{w} \otimes \mathbf{n}_{t}^{*}-\mathbf{n}_{t}^{*} \otimes \mathbf{w}+\mathbf{w} \otimes \mathbf{n}_{t}^{*}\right) \Delta \mathbf{u} \mathrm{d} \xi \mathrm{d} \eta
\end{aligned}
$$

The rearranged part is finally symmetric:

$$
\delta \Pi_{\text {press }, t}^{\circ}=-\frac{\gamma}{2} \int_{\eta} \int_{\xi} \delta \mathbf{u} \cdot\left(\mathbf{n}_{t}^{*} \otimes \mathbf{w}+\mathbf{w} \otimes \mathbf{n}_{t}^{*}\right) \Delta \mathbf{u} \mathrm{d} \xi \mathrm{d} \eta
$$

and the virtual work expression for a hydrostatic pressure load becomes after all a symmetric form in total

$$
\begin{aligned}
& \frac{\gamma}{S_{t}} \int_{\eta} \int_{\xi} \delta \mathbf{u} \cdot \mathbf{n}_{t}^{*} \mathrm{~d} \xi \mathrm{d} \eta \int_{\eta} \int_{\xi} \mathbf{n}_{t}^{*} \cdot \Delta \mathbf{u} \mathrm{d} \xi \mathrm{d} \eta \quad \text { (volume conservation) } \\
& \quad-\frac{\gamma}{2} \int_{\eta} \int_{\xi} \delta \mathbf{u} \cdot\left(\mathbf{n}_{t}^{*} \otimes \mathbf{w}+\mathbf{w} \otimes \mathbf{n}_{t}^{*}\right) \Delta \mathbf{u} \mathrm{d} \xi \mathrm{d} \eta \quad \text { (hydrostatic part) } \\
& \quad+\frac{1}{2} \int_{\eta} \int_{\xi} p_{t}\left(\begin{array}{c}
\delta \mathbf{u} \\
\delta \mathbf{u}, \xi \\
\delta \mathbf{u}, \eta
\end{array}\right) \cdot\left[\begin{array}{ccc}
0 & \underline{\mathbf{W}^{\xi}} & \underline{\mathbf{W}}^{\eta} \\
\underline{\mathbf{W}}^{\xi \mathrm{T}} & 0 & 0 \\
\underline{\mathbf{W}}^{\eta \mathrm{T}} & 0 & 0
\end{array}\right]\left(\begin{array}{c}
\Delta \mathbf{u} \\
\Delta \mathbf{u}, \xi \\
\Delta \mathbf{u}, \boldsymbol{\eta}
\end{array}\right) \mathrm{d} \xi \mathrm{d} \eta
\end{aligned}
$$

(normal change and structural displacements)

$$
=-\int_{\eta} \int_{\xi} p_{t} \delta \mathbf{u} \cdot \mathbf{n}_{t}^{*} \mathrm{~d} \xi \mathrm{d} \eta \quad \text { (load vector) }
$$

\subsection{Mixed formulation}

As an alternative a mixed formulation based on a suggestion by Taylor [18] is presented in the following with the pressure change due to the fluid level displacement $\Delta p^{\Delta \mathbf{u}_{w}}=\gamma \Delta \mathbf{u}_{w} \cdot \mathbf{w}$ for a hydrostatic pressure load as separate unknown, here written for one container only. Thus we have one equation for the volume conservation with the pressure change $\Delta p^{\Delta \mathbf{u}_{w}}$ as separate unknown, see Equation (46) and the virtual work expression with additional terms for $\Delta p^{\Delta \mathbf{u}_{w}}$, see Equation (47). The mixed formulation for the hydrostatic pressure becomes

$$
\begin{gathered}
\Delta p^{\Delta \mathbf{u}_{w}}-\frac{\gamma}{S_{t}} \int_{\eta} \int_{\xi} \mathbf{n}_{t}^{*} \cdot \Delta \mathbf{u} \mathrm{d} \xi \mathrm{d} \eta=0 \\
\Delta p^{\Delta \mathbf{u}_{w}} \int_{\eta} \int_{\xi} \delta \mathbf{u} \cdot \mathbf{n}_{t}^{*} \mathrm{~d} \xi \mathrm{d} \eta
\end{gathered}
$$




$$
\begin{aligned}
& -\frac{\gamma}{2} \int_{\eta} \int_{\xi} \delta \mathbf{u} \cdot\left(\mathbf{n}^{*} \otimes \mathbf{w}+\mathbf{w} \otimes \mathbf{n}^{*}\right) \Delta \mathbf{u} \mathrm{d} \xi \mathrm{d} \eta \\
& +\int_{\eta} \int_{\xi} \frac{p_{t}}{2}\left(\begin{array}{c}
\delta \mathbf{u} \\
\delta \mathbf{u}, \xi \\
\delta \mathbf{u}, \eta
\end{array}\right) \cdot\left[\begin{array}{ccc}
0 & \underline{\mathbf{W}}^{\xi} & \underline{\mathbf{W}}^{\eta} \\
\underline{\mathbf{W}}^{\xi \mathrm{T}} & 0 & 0 \\
\underline{\mathbf{W}}^{\eta \mathrm{T}} & 0 & 0
\end{array}\right]\left(\begin{array}{c}
\Delta \mathbf{u} \\
\Delta \mathbf{u}, \xi \\
\Delta \mathbf{u}, \boldsymbol{\eta}
\end{array}\right) \mathrm{d} \xi \mathrm{d} \eta \\
& =-\int_{\eta} \int_{\xi} p_{t} \delta \mathbf{u} \cdot \mathbf{n}_{t}^{*} \mathrm{~d} \xi \mathrm{d} \eta
\end{aligned}
$$

If these forms are taken, a different development concerning the symmetry of the expression is possible, which becomes more visible after the FE-discretization shown in the following.

\section{FE-DISCRETIZATION}

The variation of the total potential energy $W$ with $V$ as the elastic potential of the structure and $\delta \Pi$ as the virtual work of the external forces vanishes, see also Section 4.3,

$$
\delta W=\delta V-\delta \Pi=0
$$

Linearizing by means of a Taylor series expansion and assuming a pure displacement approximation, we embed the non-linear formulation in a Newton-type scheme as discussed above.

$$
\delta W\left(\mathbf{x}_{t}+\Delta \mathbf{u}\right)=\delta W\left(\mathbf{x}_{t}\right)+D \delta W\left(\mathbf{x}_{t}\right) \Delta \mathbf{u}=0
$$

Equation (49) allows to establish a piecewise linear system of equations:

$$
D\left[\delta V\left(\mathbf{x}_{t}\right)-\delta \Pi\left(\mathbf{x}_{t}\right)\right] \Delta \mathbf{u}=-\left[\delta V\left(\mathbf{x}_{t}\right)-\delta \Pi\left(\mathbf{x}_{t}\right)\right]
$$

If an isoparametic representation is taken for the FE-discretization

$$
\mathbf{x}_{t}=\mathbf{N} \mathbf{x} ; \quad \Delta \mathbf{u}=\mathbf{N d} \quad \text { and } \quad \delta \mathbf{u}=\mathbf{N} \delta \mathbf{d}
$$

then the global stiffness matrix and the load vector become

$$
\left[V,,_{\mathbf{x x}}-\Pi, \mathbf{x x}\right] \mathbf{d}=-\left(V,,_{\mathbf{x}}-\Pi, \mathbf{x}\right)
$$

Following Reference [9] and taking $\mathbf{K}_{T}=V, \mathbf{x x}$ and $\Pi, \mathbf{x x}$ from Equation (47) the coupled problem for the deformation including the volume change is then written in the case of one container $i$ as follows, if the alternative mixed formulation is used, thus including Equation 
(46) and $V,,_{\mathbf{x}}=\mathbf{f}_{\text {int }}, \Pi, \mathbf{x}=\mathbf{f}_{\text {ext }}+\mathbf{f}_{\text {press }, i}$ :

$$
\left[\begin{array}{cc}
\mathbf{K}_{T}-\mathbf{K}_{\text {press }, i} & -\mathbf{a}_{i} \\
-\mathbf{a}_{i}^{\mathrm{T}} & -b_{i}^{-1}
\end{array}\right]\left(\begin{array}{c}
\mathbf{d} \\
\Delta p^{\Delta \mathbf{u}_{w}}
\end{array}\right)=\left(\begin{array}{c}
\mathbf{f}_{\text {ext }}+\mathbf{f}_{\text {press }, i}-\mathbf{f}_{\mathrm{int}} \\
0
\end{array}\right)
$$

The matrix $\mathbf{K}_{\text {press, } i}$, the vector $\mathbf{a}_{i}$ and the scalar $b_{i}$ will be identified in the following. After elimination of the additional unknown $\Delta p^{\Delta \mathbf{u}_{w}}$ the symmetric form of the pure displacement formulation Equation (45) is achieved again

$$
\begin{aligned}
{\left[\mathbf{K}_{T}-\mathbf{K}_{\text {press }, i}+b_{i} \mathbf{a}_{i} \otimes \mathbf{a}_{i}\right] \mathbf{d}=} & \mathbf{f}_{\text {ext }}+\mathbf{f}_{\text {press, } i}-\mathbf{f}_{\text {int }} \\
\mathbf{K}_{\text {press }, i}= & \sum_{e} \int_{\eta} \int_{\xi} \frac{p_{t, i}}{2}\left(\begin{array}{c}
\mathbf{N} \\
\mathbf{N}_{\xi} \\
\mathbf{N}_{\eta}
\end{array}\right)^{\mathrm{T}}\left[\begin{array}{rrr}
0 & \underline{\mathbf{W}^{\xi}} & \underline{\mathbf{W}^{\eta}} \\
\underline{\mathbf{W}}^{\xi \mathrm{T}} & 0 & 0 \\
\underline{\mathbf{W}}^{\eta \mathrm{T}} & 0 & 0
\end{array}\right]\left(\begin{array}{c}
\mathbf{N} \\
\mathbf{N}_{\xi} \\
\mathbf{N}_{\eta}
\end{array}\right) \mathrm{d} \xi \mathrm{d} \eta \\
& -\frac{\gamma}{2} \sum_{e} \int_{\eta} \int_{\xi} \mathbf{N}^{\mathrm{T}}\left(\mathbf{n}_{t}^{*} \otimes \mathbf{w}+\mathbf{w} \otimes \mathbf{n}_{t}^{*}\right) \mathbf{N} \mathrm{d} \xi \mathrm{d} \eta \\
\mathbf{a}_{i}= & \sum_{e} \int_{\eta} \int_{\xi} \mathbf{N}^{\mathrm{T}} \mathbf{n}_{t}^{*} \mathrm{~d} \xi \mathrm{d} \eta \\
\mathbf{f}_{\text {press }, i}= & \sum_{e} \int_{\eta} \int_{\xi} p_{t, i} \mathbf{N}^{\mathrm{T}} \mathbf{n}_{t}^{*} \mathrm{~d} \xi \mathrm{d} \eta \\
b_{i}= & -\frac{\gamma}{S_{t, i}}\left(S_{t, i} \neq 0 \text { for container not completely filled }\right) \\
e & : \text { number of surface elements describing the wetted surface }
\end{aligned}
$$

The global stiffness matrix and the global load vector are obtained by adding the load stiffness matrix $\mathbf{K}_{\text {press, } i}$ and the load vector $\mathbf{f}_{\text {press, } i}$ to the element stiffness matrix $\mathbf{K}_{T}$ containing linear and non-linear terms, respectively, the residuum vector $\mathbf{f}_{\text {int }}$ and the vector of the external forces $\mathbf{f}_{\text {ext }}[11,13,17]$. The volume dependence leads to a second load stiffness part, a rankone update of the global stiffness matrix for each container $i$, thus linking of all variables describing the deformation of the wetted surface of container $i$, see also References [9, 10, 17]. If a structure consists of $k$ containers, then we get in general different pressure matrices for each container $i$ and due to the volume changes $k$ different rank-one updates have to be taken into account

$$
\left[\mathbf{K}_{T}-\sum_{i=1}^{k}\left(\mathbf{K}_{\text {press }, i}+b_{i} \mathbf{a}_{i} \otimes \mathbf{a}_{i}\right)\right] \mathbf{d}=\mathbf{f}_{\mathrm{ext}}+\sum_{i=1}^{k} \mathbf{f}_{\text {press }, i}-\mathbf{f}_{\text {int }}
$$




\section{SOLUTION ALGORITHM}

As already shown in References [9, 10,17] without restriction of any kind, Equation (54) can be rewritten for one container as follows:

$$
\left[\mathbf{K}^{*}+b \mathbf{a} \otimes \mathbf{a}\right] \mathbf{d}=\mathbf{F}
$$

with $\mathbf{K}^{*}=\mathbf{K}_{T}-\mathbf{K}_{\text {press }}$ and $\mathbf{F}=\mathbf{f}_{\text {ext }}+\mathbf{f}_{\text {press }}-\mathbf{f}_{\text {int }}$.

According to Sherman-Morrison [19] the solution of such a problem can be achieved by

$$
\begin{aligned}
\mathbf{d} & =\Delta \mathbf{u}_{\mathrm{I}}-\beta \Delta \mathbf{u}_{\mathrm{II}} \otimes \Delta \mathbf{u}_{\mathrm{II}} \mathbf{F} \\
\text { with } \quad \Delta \mathbf{u}_{\mathrm{I}} & =\mathbf{K}^{*-1} \mathbf{F} \\
\Delta \mathbf{u}_{\mathrm{II}} & =\mathbf{K}^{*-1} \mathbf{a} \\
\text { and } \quad \beta & =\frac{b}{1+b \mathbf{a} \cdot \Delta \mathbf{u}_{\mathrm{II}}}
\end{aligned}
$$

Introducing Equations (62)-(64) into Equation (61) the solution vector $\mathbf{d}$ can be obtained by one additional forward backward substitution for each container plus two scalar products within the solution process using a direct solver

$$
\mathbf{d}=\Delta \mathbf{u}_{\mathrm{I}}-\frac{b \Delta \mathbf{u}_{\mathrm{II}} \cdot \mathbf{F}}{1+b \mathbf{a} \cdot \Delta \mathbf{u}_{\mathrm{II}}} \Delta \mathbf{u}_{\mathrm{II}}
$$

It must be noted that in the input phase a specification of all loaded surface segments, their relation to closed volumes and their normal direction is necessary. Within the algorithm the actual volume of the fluid in each container and the elements wetted by the fluid as well as the fluid level has to be computed in order to get the corresponding pressure correctly. The fluid level displacement in each iteration step is computed via, see also Equation (29):

$$
\Delta u_{w}=\Delta \mathbf{u}_{w} \cdot \mathbf{w}=\frac{1}{S_{t}} \mathbf{a} \cdot \mathbf{d}
$$

If a Gauss point of a surface is observed to be wetted, then the corresponding term is included in the stiffness and force terms. Thus it may occur that surface elements are only partially wetted and the interpolation somehow disperses this effect. If a completely fluid filled structure is given, the area of the free fluid surface $S_{t, i}$ vanishes. For this case a compressible fluid under gravity has to be considered, see Reference [20].

\section{VOLUME EFFECTS ON THE STIFFNESS OF STRUCTURES}

It is physically obvious, that the linkage of all variables by an additional equation leads to a change in the stiffness of the system, indicated by a different value of the determinant and modified eigenvalues $\lambda$ of the stiffness matrix, illustrated by a simple example similar as presented in Reference [9] for gas loading, see Figure 5. A container with rigid walls and with two springs supporting the walls (spring stiffnesses $c_{1}, c_{2}$; surface of the wetted walls $A$ ) is 


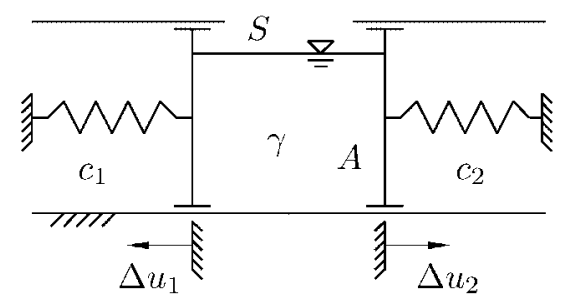

Figure 5. Stiffness effects due to volume constraints.

pressurized by a hydrostatic pressure load. For the global stiffness matrix $\mathbf{K}$, the determinant and the eigenvalues become with $b=-\gamma / S$ :

$$
\begin{aligned}
\mathbf{K} & =\left[\begin{array}{ll}
c_{1} & 0 \\
0 & c_{2}
\end{array}\right]+b\left(\begin{array}{l}
A \\
A
\end{array}\right)(A, A) \\
\operatorname{det} \mathbf{K} & =c_{1} c_{2}+b A^{2}\left(c_{1}+c_{2}\right) \\
\lambda_{1,2} & =\frac{1}{2}\left[c_{1}+c_{2}+2 b A^{2} \pm \sqrt{\left(c_{1}-c_{2}\right)^{2}+\left(2 b A^{2}\right)^{2}}\right]
\end{aligned}
$$

In the case $c_{1}=c_{2}=c$ this simplifies to

$$
\begin{aligned}
\operatorname{det} \mathbf{K} & =c^{2}+2 b A^{2} c \\
\lambda_{1} & =c ; \quad \lambda_{2}=c+2 b A^{2}
\end{aligned}
$$

The first eigenform and eigenvalue simply describes a parallel sideward motion, whereas the second eigenpair describes a counter motion against which the pressure provides a higher or lower stiffness.

\section{NUMERICAL EXAMPLES}

\subsection{Elastic hollow hook under hydrostatic pressure loading}

This example is chosen to show the effect of a hydrostatic pressure loading on an overall deforming elastic structure. The structure looking like a hollow hook is loaded by filling, thus a consistent addition of fluid (elastic modulus of the structure $E=1.0 \times 10^{5} \mathrm{~N} / \mathrm{cm}^{2}$, Poisson's ratio $v=0.3$, specific weight of the fluid $\gamma=0.1 \mathrm{~N} / \mathrm{cm}^{3}$ ), see Figure 6 . Thus with increasing fluid level the hook deforms under the pressure load, see Figure 7. Figure 8 shows the change of the fluid surface area $S$ and the displacement $\left|\mathbf{d}_{A}\right|$ of a selected point $A$ during the loading process. Furtheron in Table I the convergence rate at a fluid level of $59 \mathrm{~cm}$ is given. The rate is quadratic, indicating the advantage of a consistent linearized stiffness matrix. If for comparison only the load stiffness part is neglected only a linear convergence rate can be observed. 

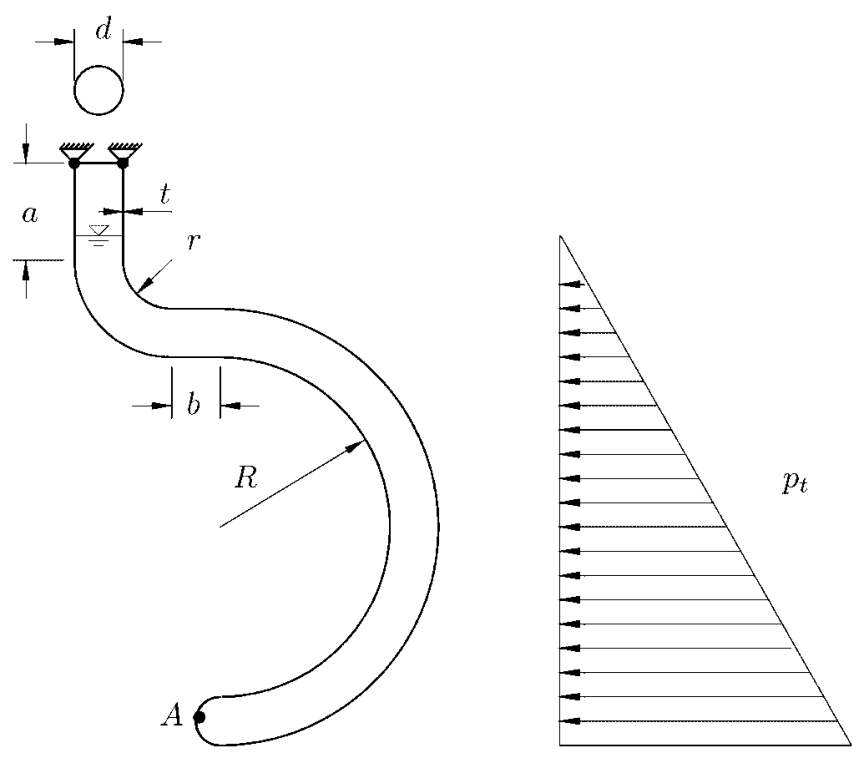

Figure 6. Elastic hollow hook under hydrostatic pressure loading, final loading state; geometrical data: $a=20 \mathrm{~cm}, b=10 \mathrm{~cm}, r=10 \mathrm{~cm}, R=25 \mathrm{~cm}, d=10 \mathrm{~cm}, t=0.2 \mathrm{~cm}$.

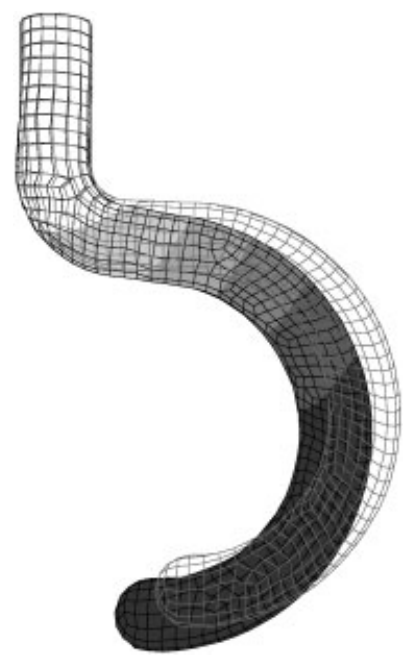

Figure 7. Elastic hollow hook under hydrostatic pressure loading; undeformed structure (meshed), deformed structure (fully filled).

\subsection{Fluid filled structure under torsion and axial loading}

This example is chosen to show the interaction of large deformations with hydrostatic pressure loading. An elastic hollow structure filled by fluid (elastic modulus of the structure 


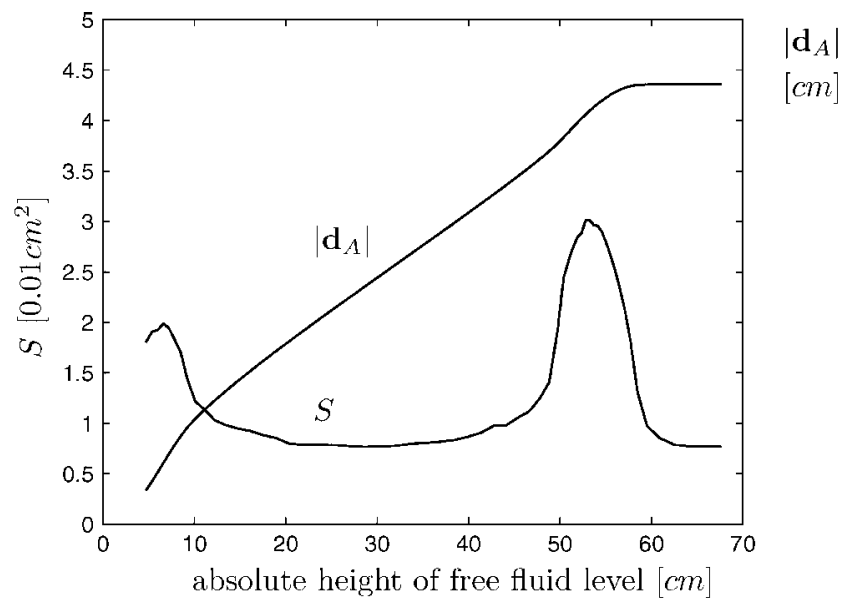

Figure 8. Elastic hollow hook under hydrostatic pressure loading; area $S$ of free fluid surface vs fluid level; displacement $\left|\mathbf{d}_{A}\right|$ of point $A$ vs fluid level.

Table I. Elastic hollow hook under hydrostatic pressure loading; convergence rate at fluid level of $59 \mathrm{~cm}$.

\begin{tabular}{lcccccc}
\hline Iteration step & 1 & 2 & 3 & 4 & 5 & 6 \\
\hline Energy & $4.65 \times 10^{-2}$ & $5.25 \times 10^{-5}$ & $1.64 \times 10^{-7}$ & $5.17 \times 10^{-10}$ & $1.62 \times 10^{-12}$ & $5.09 \times 10^{-15}$ \\
\hline
\end{tabular}

$E_{1}=0.75 \times 10^{5} \mathrm{~N} / \mathrm{cm}^{2}, E_{2}=1.0 \times 10^{6} \mathrm{~N} / \mathrm{cm}^{2}$, Poisson's ratio $v=0.3$, specific weight of the fluid $\left.\gamma=0.1 \mathrm{~N} / \mathrm{cm}^{3}\right)$ is loaded by torsion $\left(\varphi_{\text {ext }}\right)$ and axial loading $\left(u_{\text {ext }}\right)$, see Figure 9 . The base and top plates have a higher stiffness as the wall of the structure to avoid a snap through behaviour. In addition the top plate has a breather hole to get a pure hydrostatic loading within the deformation process. At the beginning of the analysis the container is partially filled with fluid (volume $V=375 \mathrm{~cm}^{3}$ ), leading to an initial fluid level $w_{0}$. With increasing deformation the fluid level $w$ increases due to the decreasing internal volume of the structure, see Figures 10, 11. Again in Tabel II the convergence rate at a torsion angle of $\varphi_{\mathrm{ext}}=15^{\circ}$ is given, showing quadratic convergence.

\subsection{Interaction of neighboured fluid levels in an elastic assembled structure}

This example is chosen to show the interaction of two fluids contained in two neighboured deforming elastic structures. The structure consists of two assembled containers (elastic modulus $E=2.0 \times 10^{4} \mathrm{~N} / \mathrm{cm}^{2}$, Poisson's ratio $v=0.3$, wall thickness $t=0.1 \mathrm{~cm}$, specific gravity of the fluid $\gamma=0.1 \mathrm{~N} / \mathrm{cm}^{3}$ ), see Figure 12. At the beginning of the analysis the left container is partially filled with a fluid with volume $V_{1}=375 \mathrm{~cm}^{3}$ indicated by an initial fluid level $w_{10}$ whereas the right container is loaded by filling from $w_{20}=0$, thus a consistent addition of fluid 


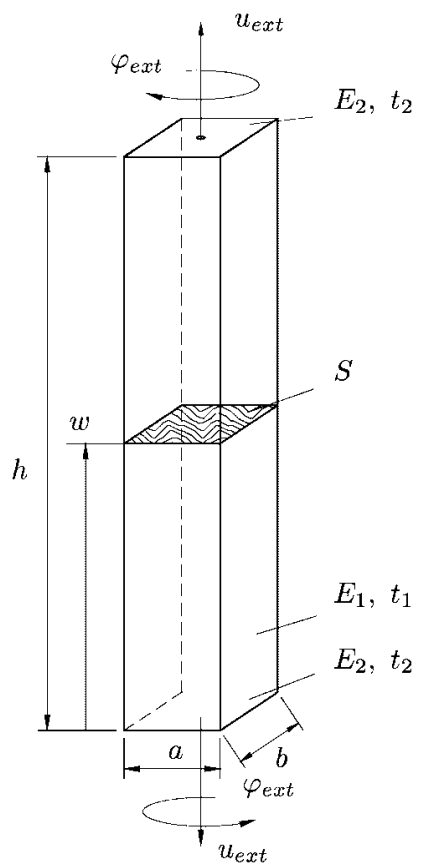

Figure 9. Fluid filled structure under torsion and axial loading; geometrical data: $a=5 \mathrm{~cm}, b=5 \mathrm{~cm}$, $h=30 \mathrm{~cm}, t_{1}=0.1 \mathrm{~cm}, t_{2}=1 \mathrm{~cm}$, fluid loading: $u_{\mathrm{ext}}=1.25 \mathrm{~cm}, \varphi_{\mathrm{ext}}=45^{\circ}$.

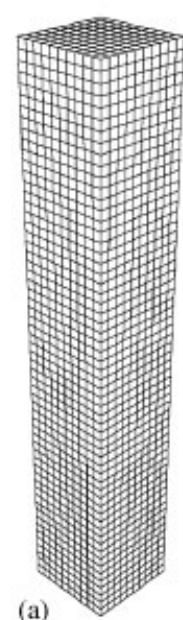

(a)

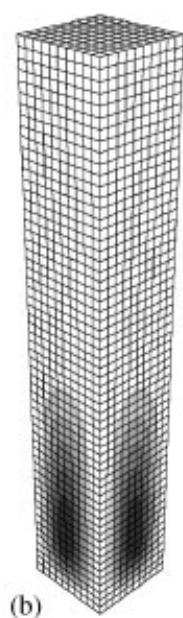

(b)

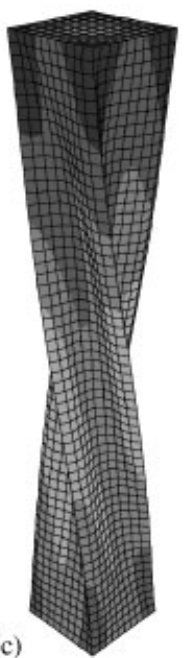

(c)

Figure 10. Fluid filled structure under torsion and axial loading: (a) undeformed structure; (b) filled structure without torsion loading; and (c) filled structure with torsion and axial loading. 


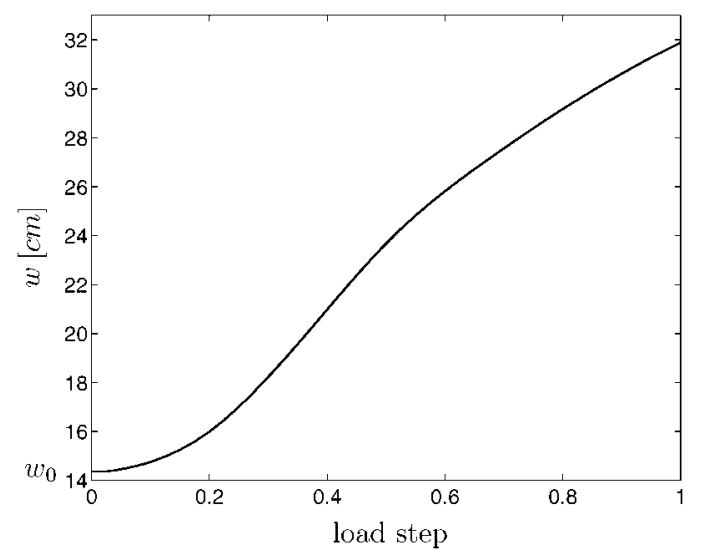

Figure 11. Fluid filled structure under torsion and axial loading; fluid level $w$ vs load step; initial fluid level $w_{0}$.

Table II. Fluid filled structure under torsion and axial loading; convergence rate at a torsion angle of $\varphi_{\mathrm{ext}}=15^{\circ}$.

\begin{tabular}{lcccccc}
\hline Iteration step & 1 & 2 & 3 & 4 & 5 & 6 \\
\hline Energy & $8.82 \times 10^{+3}$ & $1.17 \times 10^{+1}$ & $2.89 \times 10^{-3}$ & $9.76 \times 10^{-8}$ & $2.59 \times 10^{-11}$ & $8.45 \times 10^{-15}$ \\
\hline
\end{tabular}

in each load step. The structure is deformed during the loading process and as a consequence of the deformation the fluid level $w_{1}$ in the left container changes as well, see Figures 13 and 14. As in the previous examples the convergence rate is quadratic.

\section{CONCLUSIONS}

The derivations for deformation dependent loading considering a hydrostatic pressure load show that the conservativeness of structural problems can be proven when taking the volume conservation for the fluid into account by checking the symmetry of the linearized weak form of equilibrium. The particular boundary conditions have been worked out. The discretization with FE leads for each container to a rank-one update of the stiffness matrix. This special structure can be directly used to achieve a very efficient solution scheme, see also References $[9,10]$ in particular compared to a complete discretization of the fluid. Within a non-linear analysis and a Newton-type scheme using finite elements the consistent linearization of the deformation dependent terms leads to a quadratic convergence. A very important result of the derivation is that no specific fluid elements are needed to investigate the hydrostatic effect.

The numerical examples show the necessity of taking the deformation dependence for all terms, in particular, the change of the shape of the volume as well as the volume due to the loading into account. Without these terms correct results cannot be obtained. The stiffness 


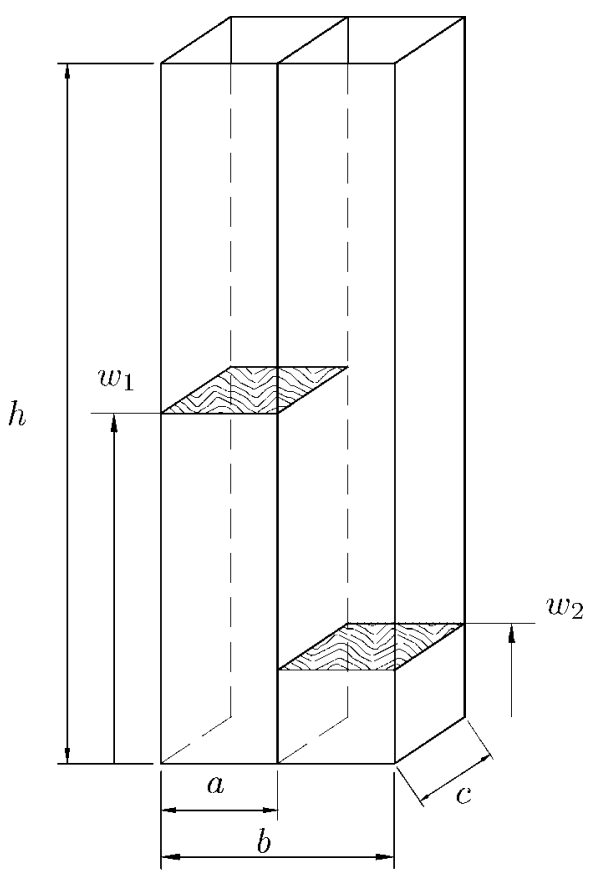

Figure 12. Interaction of neighboured fluid levels in an elastic assembled structure; geometrical data: $a=5 \mathrm{~cm}, b=10 \mathrm{~cm}, c=5 \mathrm{~cm}, h=30 \mathrm{~cm}$.

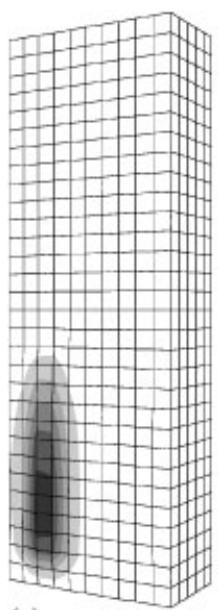

(a)

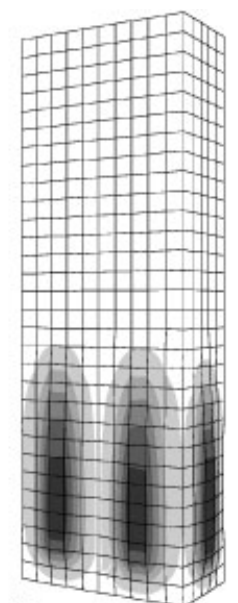

(b)



(c)

Figure 13. Interaction of neighboured fluid levels in an elastic assembled structure; status of right container: (a) empty; (b) semi charged; and (c) fully charged. 


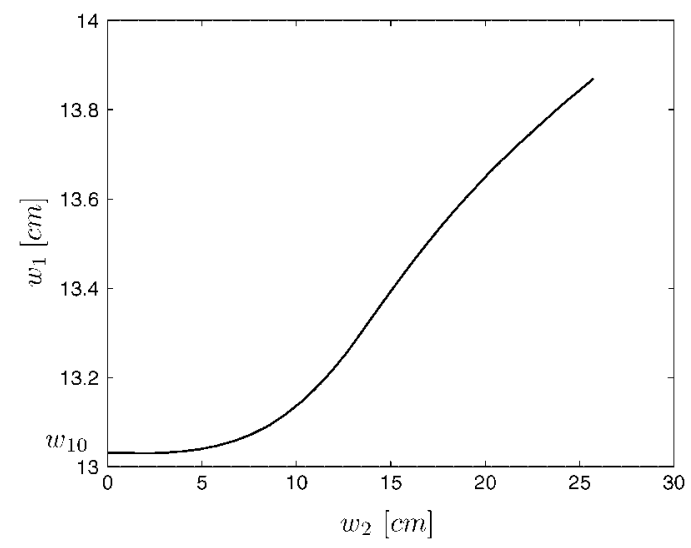

Figure 14. Interaction of neighboured fluid levels in an elastic assembled structure; fluid level $w_{1}$ of left container vs fluid level $w_{2}$ of right container, initial fluid level $w_{10}$ in the left container.

of the structures against other external loading increases with the satisfaction of the volume conservation, as a further constrained is imposed for each container under hydrostatic pressure loading.

\section{REFERENCES}

1. Pearson CE. General theory of elastic stability. Quarterly of Applied Mathematics 1956; 14:133-144.

2. Schweizerhof K. Nonlinear analysis of structures under deformation dependent loading using finite elements. Dissertation, University Stuttgart, 1982 (in German).

3. Schweizerhof K, Ramm E. Displacement dependent pressure loads in non-linear finite element analyses. Computers and Structures 1984; 18(6):1099-1114.

4. Bufler H. Pressure Loaded Structures under Large Deformations. Zeitschrift für Angewandte Mathematik und Mechanik 1984; 64(7):287-295.

5. Bufler H. Zur Potentialeigenschaft der von einer Flüssigkeit herrührenden Druckbelastung. Zeitschrift für Angewandte Mathematik und Mechanik 1985; 65(4):130-132.

6. Bufler H. Konsistente und nichtkonsistente Druckbelastungen durch Flüssigkeiten. Zeitschrift für Angewandte Mathematik und Mechanik 1992; 72(7):T172-T175.

7. Bufler H. Configuration dependent loading and non-linear elastomechanics. Zeitschrift für Angewandte Mathematik und Mechanik 1993; 73:4-5.

8. Schneider H. Fluid loaded membranes undergoing large deformations. Dissertation, University Stuttgart, 1990 (in German).

9. Rumpel T, Schweizerhof K. Volume dependent pressure loading and its influence on the stability of structures. International Journal for Numerical Methods in Engineering 2003; 56:211-238.

10. Bonet J, Wood RD, Mahaney J, Heywood P. Finite element analysis of air supported membrane structures. Computer Methods in Applied Mechanics and Engineering 2000; 190:579-595.

11. Rumpel T, Schweizerhof K. Volumenabhängige Druckbelastung bei großen Deformationen. Proceedings in Applied Mathematics and Mechanics 2002; 1(1):232-233.

12. Rumpel T, Schweizerhof K. Volumenabhängige hydrostatische Druckbelastung in der nichtlinearen FE-Analyse. Proceedings in Applied Mathematics and Mechanics 2003; 2(1):256-257.

13. Rumpel T, Schweizerhof K. A hybrid approach for volume dependent fluid-structure-problems in non-linear static finite element analysis. In Proceedings of the Fifth World Congress on Computational Mechanics (WCCM V), Mang HA, Rammerstorfer FG, Eberhardsteiner J (eds), Vienna, Austria 7-12 July, 2002.

14. Romano G. Potential operators and conservative systems. Meccanica 1972; 7:141-146. 
15. Sewell MJ, On Configuration-Dependent Loading. Archive fur Rational Mechanics and Analysis 1966; 23: $327-351$

16. Knebel K. Stability of steel cylinders with unilateral boundary conditions under static and dynamic loading. Dissertation, University Karlsruhe, 1997 (in German).

17. Schweizerhof K, Rumpel T. Volume dependent pressure loading and large deformation finite element analysis of structures. In Trends in Computational Structural Mechanics, Wall WA, Bletzinger K-U, Schweizerhof K (eds), International Center for Numerical Methods in Engineering (CIMNE), Barcelona, Spain, 2001; $386-396$

18. Taylor RL. Department of Civil and Environmental Engineering, University of California Berkeley, personal communication, 2001.

19. Zielke H. Inversion of modified symmetric matrices. Journal of the Association for Computing Machinery 1968; 15:402-408.

20. Rumpel T, Schweizerhof K. On an efficient model for gas and fluid supported membranes and shell structures. In Textile Composites and Inflatable Structures, Oñate E, Kröplin B (eds). International Center for Numerical Methods in Engineering (CIMNE), Barcelona, Spain, 2003.

21. Hauptmann R, Schweizerhof K. A systematic development of solid-shell element formulations for linear and non-linear analyses employing only displacement degrees of freedom. International Journal for Numerical Methods in Engineering 1998; 42:49-69. 